\title{
Improper colouring of (random) unit disk graphs $^{\dagger}$
}

\author{
Ross J. Kang ${ }^{1 \ddagger}$ Tobias Müller ${ }^{1 \S}$ and Jean-Sébastien Sereni ${ }^{2 \uparrow}$ \\ ${ }^{1}$ Oxford University, Department of Statistics, 1 South Parks Road, Oxford OX1 3TG, United Kingdom \\ ${ }^{2}$ MASCOTTE, I3S-CNRS/INRIA/UNSA, 2004 Route des Lucioles, BP93, F-06902, Sophia-Antipolis, France
}

For any graph $G$, the $k$-improper chromatic number $\chi^{k}(G)$ is the smallest number of colours used in a colouring of $G$ such that each colour class induces a subgraph of maximum degree $k$. We investigate the ratio of the $k$ improper chromatic number to the clique number for unit disk graphs and random unit disk graphs to extend results of McDiarmid and Reed (1999); McDiarmid (2003) (where they considered only proper colouring).

Keywords: improper colouring, unit disk graphs, random unit disk graphs, radio channel assignment

\section{Introduction}

Given a set $V$ of points in the plane and a distance threshold $d>0$, we let $G(V, d)$ denote the following graph. The vertex set is $V$ and distinct vertices form an edge whenever the Euclidean distance between them is less than $d$. Any graph isomorphic to such a graph is called a unit disk graph. The study of the class of unit disk graphs stems partly from applications in communication networks. In particular, the problem of finding a proper vertex-colouring - in which the vertices of a graph are coloured so that adjacent vertices do not receive the same colour - of a given unit disk graph is closely associated (due to Hale (1980)) with the so-called frequency allocation problem. Consult Leese and Hurley (2002) for a more general treatment of this important problem.

McDiarmid and Reed (1999) and McDiarmid (2003) investigated the chromatic number $\chi$ for unit disk graphs in two related cases. The first case is the asymptotic limit of $\chi$ as the distance threshold $d$ approaches infinity and $V$ is infinite. Given the motivation of frequency allocation, it is reasonable then to suppose that $V$ is big and the points are well distributed in the plane. McDiarmid and Reed (1999) show that, for sets $V$ with finite upper density, the ratio of chromatic number over clique number approaches $2 \sqrt{3} / \pi$ as $d \rightarrow \infty$. The second case is the asymptotic behaviour of $\chi$ for unit disk graphs based on randomly chosen points in the plane (where the distance threshold $d$ approaches 0 as the number

\footnotetext{
${ }^{\dagger}$ This work was partially supported by Région Provence-Alpes-Côte D’Azur.

¥This author is partially supported by NSERC of Canada and the Commonwealth Scholarship Commission (UK).

$\S$ This author is partially supported by EPSRC, the Department of Statistics, Bekker-la-Bastide fonds, Dr. Hendrik Muller's Vaderlandsch fonds, and Prins Bernhard Cultuurfonds

๑ This author is partially supported by the European project FET-CRESCCO.
} 
of points $n$ approaches infinity). The paper McDiarmid (2003) establishes almost sure (and in probability) convergence results for these random instances of unit disk graphs.

In this paper, we are also interested in vertex-colourings of unit disk graphs; however, we partially relax the condition that any two vertices with the same colour may not be adjacent. Recall that, given an arbitrary colouring, a colour class is a set of vertices all assigned the same colour. Given $k \geq 0$, we say that a graph is $k$-improper colourable if there is a colouring in which each colour class induces a subgraph with maximum degree at most $k$. We wish to find the minimum number $\chi^{k}$ of colours used in such a colouring. Note that proper colouring is just 0-improper colouring and hence $\chi=\chi^{0}$. Our aim, in this paper, is to determine $\chi^{k}$ for unit disk graphs in the two cases mentioned above.

Improper colourings are studied both for theoretical and practical interests. For instance, they can be used to model computing shared resources where the number of users per shared resource is limited. In terms of communication systems, our precise motivation is the following problem, posed by Alcatel Industries: a communications satellite directs signals at several receivers on the Earth, each of which listens on a chosen frequency. Of course, we wish to minimise the number of channels used. There is a limit to how focused upon its target each signal can be; indeed, nearby receivers on the same frequency will experience interference (causing noise in the signal). Our assumptions are that noise is a symmetric contribution (i.e. if the signal for receiver $u$ contributes noise to the signal for receiver $v$, then vice versa) and that the intensity of noise caused by a nearby signal is independent of the frequency and receiver. We will also assume in this situation that each receiver can tolerate some threshold $k$ of noise. We define a noise graph: the vertices are the receivers and we put an edge between $u$ and $v$ if $u$ is in the noise area of $v$ (and $v$ in the noise area of $u$ ). The frequencies are represented by colours. So an assignment of frequencies to receivers is equivalent to a $k$-improper colouring of the noise graph. We aim at minimising the number of colours (i.e. frequencies) used. The reader can refer to Havet and Sereni (2005) for more details and other study of this problem.

Before going further, we must review and introduce some basic terminology. We denote the maximum degree of $G$ by $\Delta(G)$. A clique is a set of pairwise adjacent vertices; the clique number $\omega(G)$ is the supremum of the numbers of vertices in a clique of $G$. It is clear that $\chi^{k}(G) \geq \omega(G) /(k+1)$. We also note the following proposition, which is a corollary of a result due to Lovász (1966).

Proposition 1 For any graph $G, \chi^{k}(G) \leq\left\lceil\frac{\Delta(G)+1}{k+1}\right\rceil$.

We note that the clique number of a unit disk graph can be found in polynomial time by means of an $\mathrm{O}\left(n^{4.5}\right)$ algorithm (Clark et al. (1990)) and even when an explicit representation in the plane is not available (Raghavan and Spinrad (2003)). In contrast, the problem of finding the chromatic number of unit disk graphs is NP-complete (Clark et al. (1990)). Recent work in Havet et al. (2005) shows that the same holds for the $k$-improper chromatic number $\chi^{k}$, for any fixed $k$. Furthermore, by the above proposition and since $\Delta(G) \leq 6 \omega-6$ for any unit disk graph $G$ (to see this, consider that each $\pi / 3$-sector of a unit disk induces a clique), we have a heuristic for $\chi^{k}$ with approximation ratio 6 .

\section{Asymptotically, improperly colouring unit disk graphs}

This section discusses our extensions of McDiarmid and Reed (1999). Let $V$ be any countable set of points in the plane. For $x>0$, let $f(x)$ be the supremum of the ratio $|V \cap S| / x^{2}$ over all open $(x \times x)$ squares $S$ with sides aligned with the axes. The upper density of $V$ is $\sigma^{+}(V)=\inf _{x>0} f(x)$. 
Theorem 1 (McDiarmid and Reed (1999)) Let $V$ be a countable non-empty set of points in the plane with upper density $\sigma^{+}(V)=\sigma$. Then $\omega(G(V, d)) / d^{2} \geq \sigma \pi / 4$ and $\chi(G(V, d)) / d^{2} \geq \sigma \sqrt{3} / 2$ for any $d>0$; and, as $d \rightarrow \infty$, we have $\Delta(G(V, d)) / d^{2} \rightarrow \sigma \pi, \omega(G(V, d)) / d^{2} \rightarrow \sigma \pi / 4$ and $\chi(G(V, d)) / d^{2} \rightarrow$ $\sigma \sqrt{3} / 2$.

Note that $2 \sqrt{3} / \pi \approx 1.103$. We extend this theorem as follows.

Theorem 2 Let $V$ be a countable non-empty set of points in the plane with upper density $\sigma^{+}(V)=\sigma$. Then $\chi^{k}(G(V, d)) / d^{2} \geq \frac{\sigma \sqrt{3} / 2}{k+1}$ for any $d>0$ and, as $d \rightarrow \infty$, we have $\chi^{k}(G(V, d)) / d^{2} \rightarrow \frac{\sigma \sqrt{3} / 2}{k+1}$.

In particular, for any countable set $V$ of points in the plane with finite positive upper density, the ratio of $\chi^{k}(G(V, d))$ to $\omega(G(V, d)) /(k+1)$ tends to $2 \sqrt{3} / \pi$ as $d$ approaches infinity.

McDiarmid and Reed also tighten the upper bounds in Theorem 1 for the case where the points are approximately uniformly spread over the plane. Given a set $V$ of points in the plane, a cell structure of $V$ with density $\sigma$ and radius $r$ is a family $\left(C_{v}: v \in V\right)$ of sets that partition the plane and such that each $C_{v}$ has area $1 / \sigma$ and is contained in a ball of radius $r$ about $v$.

Theorem 3 (McDiarmid and Reed (1999)) Let the set $V$ of points in the plane have a cell structure with density $\sigma$ and radius $r$. Then, for any $d>0$, we have $\omega(G(V, d)) \leq(\sigma \pi / 4)(d+2 r)^{2}$ and $\chi(G(V, d))<$ $\left((\sigma \sqrt{3} / 2)^{1 / 2}(d+2 r)+(2 / \sqrt{3})+1\right)^{2}$. Thus, (combined with Theorem 1,$) \omega(G(V, d))=(\sigma \pi / 4) d^{2}+\mathrm{O}(d)$ and $\chi(G(V, d))=(\sigma \sqrt{3} / 2) d^{2}+\mathrm{O}(d)$ as $d \rightarrow \infty$.

We extend this theorem as follows.

Theorem 4 Let the set $V$ of points in the plane have a cell structure with density $\sigma$ and radius $r$. Then, for any $d>0$,

$$
\begin{aligned}
\chi^{k}(G(V, d))<\left((\sigma \sqrt{3} / 2)^{1 / 2}(d+2 r)+(2 / \sqrt{3})+1\right) \\
\left((\sigma \sqrt{3} / 2)^{1 / 2}(d+2 r)+(2 / \sqrt{3})+2 k+1\right) /(k+1) .
\end{aligned}
$$

Thus, $\chi^{k}(G(V, d))=(\sigma \sqrt{3} / 2) d^{2} /(k+1)+\mathrm{O}(d)$ as $d \rightarrow \infty$.

The key to all of the above theorems is the special case when $V$ is the triangular lattice $T$, which is defined as the integer linear combination of the vectors $(1,0)$ and $(1 / 2, \sqrt{3} / 2)$. Let $G_{T}$ denote the graph whose vertex set is $T$ and two vertices adjacent whenever they are at distance 1 from each other. Note that the Dirichlet-Voronoï cells of the set $T$ constitute a cell structure with density $2 / \sqrt{3}$ and radius $1 / \sqrt{3}$ and, hence Theorem 4 above gives good bounds on $\chi^{k}(G(V, d))$. However, we can obtain better results, and, indeed, for $k=0$, there is an exact result.

For any $d>0$, let $\hat{d}$ be the minimum distance between two points in $T$ subject to that distance being at least $d$ (i.e. $\hat{d}$ is the least value of $\left(x^{2}+x y+y^{2}\right)^{1 / 2}$ greater than or equal to $d$ so that $x, y$ are non-negative integers). Note that $d \leq \hat{d} \leq\lceil d\rceil$, and the value of $\hat{d}^{2}$ can be computed in $\mathrm{O}(d)$ arithmetic operations.

Theorem 5 (McDiarmid and Reed (1999)) For any $d>0, \chi(G(T, d))=\hat{d}^{2}$.

Consult McDiarmid and Reed (1999) for the origin of this result. Unfortunately, when we consider $k$-improper colouring, we do not obtain an exact result such as Theorem 5, but we have a good bound:

Theorem 6 For any $d>0$,

$$
\chi^{k}(G(T, d)) \leq(\lceil d\rceil+1)\left(\left\lceil\frac{\lceil d\rceil-2}{k+1}\right\rceil+1\right)<\frac{(d+1)(d+2 k+1)}{k+1} .
$$




\section{Improper colouring of random unit disk graphs}

This section discusses our extensions of McDiarmid (2003). We consider graphs $G_{n}$ obtained as follows. We pick vertices $X_{1}, \ldots, X_{n} \in \mathbb{R}^{2}$ at random (i.i.d. according to some probability distribution $\nu$ on $\mathbb{R}^{2}$ ) and we set $G_{n}=G\left(\left\{X_{1}, \ldots, X_{n}\right\}, d(n)\right)$, where we assume we are given a sequence of distances $d(n)$ that satisfies $d(n) \rightarrow 0$ as $n \rightarrow \infty$. We will allow any choice of $\nu$ that has a bounded density. We are interested in the behaviour of the clique number, the chromatic number, and the $k$-improper chromatic number of $G_{n}$ as $n$ grows large. The distance $d(n)$ plays a role similar to that of $p(n)$ in the Erdös-Renyi random graphs $G(n, p)$. Depending on the choice of $d(n)$, qualitatively different types of behaviour can be observed. We prefer to describe the various cases in terms of the quantity $d^{2} n$, because $d^{2} n$ can be considered a measure of the average degree of the graph. Intuitively, this should be obvious (consider for instance the case $\nu$ is uniform on $[0,1]^{2}$, so that the probability of an edge between $X_{1}$ and $X_{2}$ is $\approx \pi d^{2}$ when $d$ is small and the expected degree of $X_{1}$ is therefore $\approx \pi(n-1) d^{2}$ ). For a somewhat more rigorous treatment of the relationship between $d^{2} n$ and the average degree, see McDiarmid and Müller.

Theorem 7 The following holds for proper colouring of random unit disk graphs:

1. [McDiarmid and Müller] If $d^{2} n$ is $o(\ln n)$, then $\chi\left(G_{n}\right) / \omega\left(G_{n}\right) \rightarrow 1$ almost surely.

2. [McDiarmid (2003)] If $d^{2} n / \ln n \rightarrow \infty$, then $\chi\left(G_{n}\right) / \omega\left(G_{n}\right) \rightarrow 2 \sqrt{3} / \pi$ almost surely.

Since $d^{2} n$ can be correlated with average degree, we refer to part 1 of this theorem as the "sparse" case and part 2 the "dense" case. We remark that expressions for $\chi$ not involving $\omega$ (and vice versa) can also be obtained but are less visually pleasing. Theorem 5 can be used to prove part 2 of Theorem 7 and, perhaps not suprisingly, Theorem 6 readily extends the proof to get the following result

Theorem 8 If $d^{2} n / \ln n \rightarrow \infty$, then $\chi^{k}\left(G_{n}\right) /\left\{\omega\left(G_{n}\right) /(k+1)\right\} \rightarrow 2 \sqrt{3} / \pi$ almost surely.

In the sparse case, we need to be a little bit more careful in extending the results. The proof of part 1 of Theorem 7 treats the case when $d^{2} n=o(\ln n)$ and $d^{2} n=n^{o(1)}$ (meaning that $d^{2} n=n^{a(n)}$ with $a(n) \rightarrow 0$ ) separately. In fact, in McDiarmid (2003) and Penrose (2003), only this case was considered. In this case, the results again extend to improper colouring in a straightforward manner:

Theorem 9 If $d^{2} n=o(\ln n)$ and $d^{2} n=n^{o(1)}$, then $\chi^{k}\left(G_{n}\right) /\left\{\omega\left(G_{n}\right) /(k+1)\right\} \rightarrow 1$ almost surely.

To also cover the case where $d^{2} n=o\left(n^{-\alpha}\right)$ where $\alpha>0$, the work of McDiarmid and Müller employs Brooks' lemma together with the fact that, in this case, it holds with probability one that, for all but finitely many $n$, the maximum degree $\Delta\left(G_{n}\right)$ is either equal to the clique number $\omega\left(G_{n}\right)$ or to $\omega\left(G_{n}\right)-1$ (see also Theorems 6.1 and 6.3 of Penrose (2003)), showing that in fact $\chi\left(G_{n}\right)=\omega\left(G_{n}\right)$ (for all but finitely many $n$, with probability one). For $k$-improper colouring, no analogue of Brooks' theorem exists, but we do know that $\left\lceil\frac{\omega}{k+1}\right\rceil \leq \chi^{k} \leq\left\lceil\frac{\Delta+1}{k+1}\right\rceil$ as pointed out in the introduction. The mentioned fact about the difference of the clique number and maximum degree now translates into:

Theorem 10 If $d^{2} n=o\left(n^{-\alpha}\right)$ for some $\alpha>0$, then

$$
\mathbb{P}\left(\chi^{k}\left(G_{n}\right) \in\left\{\left\lceil\frac{\omega\left(G_{n}\right)}{k+1}\right\rceil,\left\lceil\frac{\omega\left(G_{n}\right)}{k+1}\right\rceil+1\right\} \text { for all but finitely many } n\right)=1 .
$$


In fact, one can use Corollary 3.4 of Penrose (2003) to show that, if $d^{2} n \sim n^{-\frac{1}{m(k+2)}}$ with $m \in \mathbb{N}$, then $\mathbb{P}\left(\chi^{k}\left(G_{n}\right)=\left\lceil\frac{\omega\left(G_{n}\right)}{k+1}\right\rceil\right) \rightarrow c$ for some $0<c<1$. So Theorem 10 is in a sense best possible.

Using the same methods, it can be shown that, when $d^{2} n$ is bounded above by a negative power of $n$, the probability measure of $\chi^{k}$ becomes concentrated on two consecutive integers as $n$ grows large (in the sense that $\mathbb{P}\left(\chi^{k} \in\{m(n), m(n)+1\}\right) \rightarrow 1$ for some sequence $\left.m(n)\right)$. This phenomenon is dubbed focusing in Penrose $(2002,2003)$ and is well known to occur for various graph parameters in Erdös-Renyi random graphs. Recently, one of the authors (Müller) proved a conjecture of Penrose stating that when $d^{2} n=o(\ln n)$ the clique number becomes focused and the same was shown to hold for the chromatic number. The proof can be easily adapted to also yield the following

Theorem 11 If $d^{2} n=o(\ln n)$, then there exists a sequence $m(n)$ such that

$$
\mathbb{P}\left(\chi^{k}\left(G_{n}\right) \in\{m(n), m(n)+1\}\right) \rightarrow 1 .
$$

\section{Conclusion}

In Section 2, we studied the asymptotic behaviour of $\chi^{k}$ when $d \rightarrow \infty$ and $V$ is infinite. For these results, the bound in Theorem 6 suffices; however, we would be interested to know an exact expression for $\chi^{k}(G(T, d))$ for any $d$.

In Section 3, we studied the $k$-improper chromatic number of random unit disk graphs in the sparse and dense cases. Work on the intermediary case is ongoing (McDiarmid and Müller) although it appears that results there will extend to improper colouring; indeed, the results on the case when $d^{2} n=\Theta(1)$ can be extended to show an almost sure upper bound of $2 \sqrt{3} / \pi$ for $\lim _{\sup _{n \rightarrow \infty}} \chi^{k}\left(G_{n}\right) /\left\{\omega\left(G_{n}\right) /(k+1)\right\}$. Thus, for randomly generated instances $G_{n}$, the polynomial computable value $\omega\left(G_{n}\right) /(k+1)$ multiplied by the factor $2 \sqrt{3} / \pi$ (which is quite smaller than 6 ) is a reasonable approximation for the $k$-improper chromatic number when $n$ is large enough. Of course, a very important issue that we have not discussed in this respect is the rates of convergence associated with the various results in Section 3.

\section{References}

B. N. Clark, C. J. Colbourn, and D. S. Johnson. Unit disk graphs. Discrete Math., 86(1-3):165-177, 1990.

W. K. Hale. Frequency assignment: Theory and applications. IEEE Proceedings, 68(12):1497-1514, dec 1980.

F. Havet, R. J. Kang, and J.-S. Sereni. Improper colouring of unit disk graphs. In Proceedings of the 7 th International Conference on Graph Theory, Electronic Notes in Discrete Mathematics. Elsevier, September 2005. To appear.

F. Havet and J.-S. Sereni. Channel assignment and improper choosability of graphs. In Proceedings of the 31st Workshop on Graph-Theoretic Concepts in Computer Science (WG'05), Lecture Notes in Computer Science. Springer Verlag, June 2005. To appear.

R. Leese and S. Hurley, editors. Methods and Algorithms for Radio Channel Assignment. Oxford Lecture Series in Mathematics and Its Applications. Oxford University Press, Oxford, 2002.

L. Lovász. On decompositions of graphs. Studia Sci. Math. Hungar., 1:237-238, 1966. 
C. J. H. McDiarmid. Random channel assignment in the plane. Random Structures Algorithms, 22(2): 187-212, 2003.

C. J. H. McDiarmid and T. Müller. On the chromatic number of random geometric graphs. Manuscript.

C. J. H. McDiarmid and B. Reed. Colouring proximity graphs in the plane. Discrete Math., 199(1-3): 123-137, 1999.

T. Müller. On the distribution of the clique number and chromatic number of random geometric graphs. Manuscript.

M. D. Penrose. Focusing of the scan statistic and geometric clique number. Adv. in Appl. Probab., 34(4): 739-753, 2002.

M. D. Penrose. Random Geometric Graphs. Oxford University Press, Oxford, 2003.

V. Raghavan and J. Spinrad. Robust algorithms for restricted domains. J. Algorithms, 48(1):160-172, 2003. Twelfth Annual ACM-SIAM Symposium on Discrete Algorithms (Washington, DC, 2001). 